\title{
Photonic crystal fibres and effective index approaches
}

Riishede, Jesper; Libori, Stig E. Barkou; Bjarklev, Anders Overgaard; Broeng, Jes; Knudsen, Erik

\section{Published in:}

Proceeding of Optical Communication

Link to article, DOI:

10.1109/ECOC.2001.989089

Publication date:

2001

Document Version

Publisher's PDF, also known as Version of record

Link back to DTU Orbit

Citation (APA):

Riishede, J., Libori, S. E. B., Bjarklev, A. O., Broeng, J., \& Knudsen, E. (2001). Photonic crystal fibres and effective index approaches. In Proceeding of Optical Communication (Vol. 4, pp. 522-523)

https://doi.org/10.1109/ECOC.2001.989089

\section{General rights}

Copyright and moral rights for the publications made accessible in the public portal are retained by the authors and/or other copyright owners and it is a condition of accessing publications that users recognise and abide by the legal requirements associated with these rights.

- Users may download and print one copy of any publication from the public portal for the purpose of private study or research.

- You may not further distribute the material or use it for any profit-making activity or commercial gain

- You may freely distribute the URL identifying the publication in the public portal 


\title{
Photonic Crystal Fibres and effective index approaches
}

\author{
Jesper Riishede, Stig E Barkou Libori, Anders Bjarklev, Jes Broeng, and Erik Knudsen \\ Research Center COM, Technical University of Denmark, DTU, Building 345v, DK-2800 Kgs. Lyngby \\ E-mail: riishede@com.dtu.dk
}

Abstract: Photonic crystal fibres are investigated with an effective index approach. The effective index of both core and cladding is found to be wavelength dependent. Accurate modelling must respect the rich topology of these fibres.

\section{Introduction:}

Photonic Crystal Fibres (PCFs) are pure silica fibres with numerous airholes in the cladding region (see the inset of Fig. 1). In the most simple case (PCFs guiding by an effect termed modified Total Internal Reflection) the cladding airholes act to lower the effective index of the fundamental space filling mode below the refractive index of silical. This allows modes to be guided strictly along the pure silica core, since the mode is unable to couple to any cladding modes due to the index step between the guided modes and the cladding modes.

The effective cladding index of a PCF is strongly wavelength dependent. This is due to the field avoiding the airholes at short wavelengths (resulting in a cladding index approaching the refractive index of silica at short wavelengths), while the field intensity inside the airholes may be significant at long wavelengths, resulting in a cladding index significantly below that of silica. This wavelength dependent cladding index offers great flexibility in the design of PCFs, including the possibility of highly nonlinear fibres with an effective mode area of only $1 \mu \mathrm{m}^{2}$ at $1550 \mathrm{~nm}$ wavelength ${ }^{2}$, PCFs with a zero dispersion wavelength ${ }^{3}$ considerably below $1300 \mathrm{~nm}$ and endlessly singlemode PCFs (supporting only one guided mode at all wavelengths). This last possibility arises since the reduced index contrast between core and cladding with increasing frequency, ensures that the effective frequency, $V_{\text {eff }}$, remains finite at all wavelengths ${ }^{4}$. The effective frequency is given by

$$
V_{e f f}=k \rho \sqrt{n_{c o}^{2}-n_{c l}^{2}}
$$

where $n_{c o}$ is the effective core index, $n_{c l}$ is the effective cladding index, $\mathrm{k}$ is the wavenumber and $\rho$ is the core radius.

The realization of the potential of PCFs is not only a manufacturing challenge. The modelling of PCFs is also more challenging than modelling of standard step index fibres. A large number of tools for modelling PCFs have been suggested in the past. Many methods have employed full vectorial mode-solvers, including the plane wave method $^{5}$ that we employ in this paper. Other vectorial models $^{6}$ have used localised functions to describe the guided modes. The finite element $\operatorname{method}^{7}$, a multipole method $^{8}$, and a biorthonormal method have also been suggested. The simplest approach for modelling PCFs, however, treats the PCF as a step index fibre (SIF) with a strong wavelength dependence of the cladding index ${ }^{4}$. This paper investigates some of the challenges faced when using this very simple approach for modelling PCFs.

\section{PCFs and effective indices}

The wavelength dependance of the effective cladding index of a PCF is illustrated in Fig. 1. In this specific example the hole-diameter, $\mathrm{d}$, is $50 \%$ of the interhole distance, $\Lambda$. The effective index, $\beta / \mathrm{k}$, is illustrated as a function of the normalized frequency, $\Lambda / \lambda$. Here $\beta$ is the propagation constant, $\mathrm{k}$ is the free space wavenumber, and $\lambda$ is the free space wavelength. As explained above the effective index of the cladding approaches the refractive index of silica $\left(\mathrm{n}_{\mathrm{si}}=1.45\right.$ in the calculations) when the structural size, $\Lambda$, is large compared to the wavelength. On the other hand one must have a small $\Lambda$, if the possibility of a significantly reduced effective cladding index is to be employed for e.g. non-linear purposes.

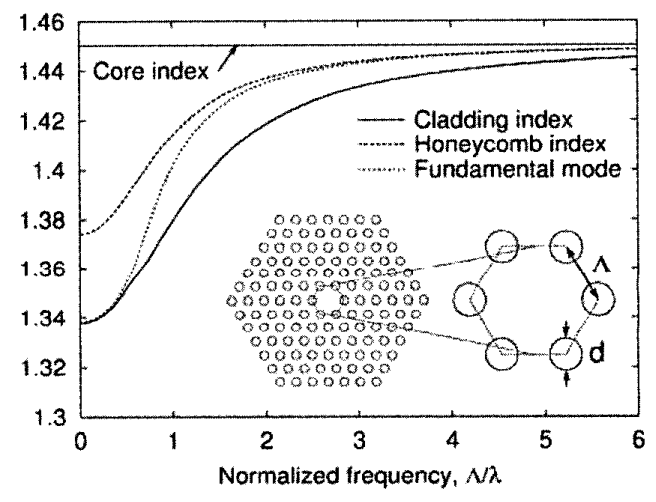

Figure 1: The effective cladding index, the corresponding honeycomb-index, the index of the fundamental mode, and the silica index. The inset shows the schematic design of a PCF, and the central honeycomb core-region.

The effective index approach assumes that a PCF may be modelled by assuming the fiber to be a step index fibre with the wavelength dependent cladding index calculated by using e.g. a plane wave method. Here, an analytical fullvectorial solution ${ }^{10}$ (no weakly-guiding approximation) to the step-index fibre problem is used, to account for the large index contrast present at long wavelengths. Since no clearly defined boundary between core and cladding exist in PCFs, this leaves some ambiguity concerning the definition of the core radius ${ }^{4}$ and the effective core-index in a SIF analogy. The refractive index of silica was used as the effective core-index in Birks et. $\mathrm{al}^{4}$. Other choices of 
core index exist, however. Since the central core-region may be seen as a honeycomb structure, as indicated in the inset of Fig. 1, we may also choose the index of the corresponding periodic honeycomb structure as the coreindex. In Fig. 1 is also shown the effective index of the fundamental space-filling mode of a honeycomb structure with the same air-hole diameter as the cladding structure. The mode-index of the fundamental guided mode is seen to approach the honeycomb index when the wavelength is short.

To analyse the accuracy of different effective index approaches, we choose in Fig. 2 to depict the normalised propagation constant,

$$
B=\frac{n_{\text {mode }}-n_{c l}}{n_{c o}-n_{c l}}
$$

as a function of $V_{\text {eff: }} B=1$ then corresponds to a mode with $\mathrm{n}_{\text {mode }}=\mathrm{n}_{\text {core }}$, while $\mathrm{B}=0$ corresponds to $\mathrm{n}_{\text {mode }}=\mathrm{n}_{\mathrm{cl}}$. $V_{\text {eff }}$ is chosen to better identify the cut-off properties. The solid lines show the calculated results for a $P C F$ with $d=0.8 \Lambda$, when $n_{\text {core }}=n_{\text {si }}$ and $\rho=\Lambda$. The maximum value of $B$ is noticed to be approximately 0.88 . The second order mode cut-off is seen to occur at $V_{c f f} \approx 4.2$, clearly in contradiction with the expected cut-off for a SIF at $V_{e f f} \approx 2.405$. This is remedied in the third example shown, by setting $\rho=0.58 \mathrm{~A}$. We then performed similar calculations for a SIF with the same parameters $\left(n_{\text {core }}=n_{s i}\right.$ $\rho=\Lambda$ and $n_{\mathrm{cl}}$ found by our plane wave method). The result is shown by the second set of curves. Resonably good agreement with the results for the PCF is seen, and we deduce that one may obtain a fair assessment of the fibre parameters by using such an approach. However, there is still some discrepancy: unrealisticly large values of $V_{\text {eft }}$ are found possible with the SIF-approximation. This could be remedied by enforcing that $V_{\text {efr }}$ should be smaller than $0.58 \times V_{e f f \text { max }}$.

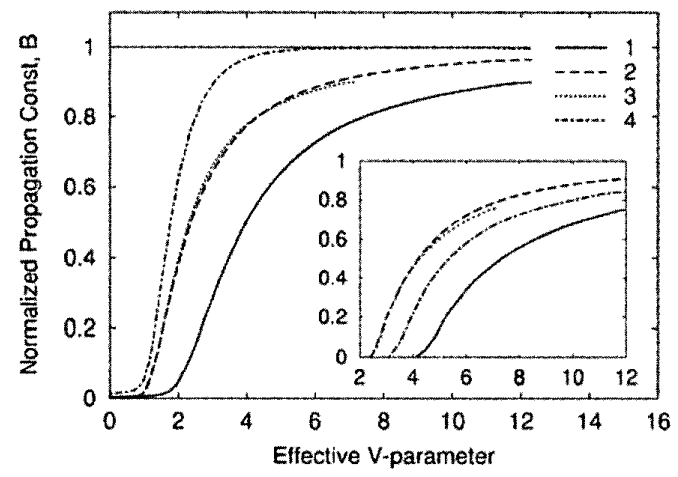

Figure 2: $B\left(V_{\text {erf }}\right)$ of the fundamental and the second order mode for 1) PCF with $n_{\mathrm{co}}=n_{\mathrm{si}}, \rho=\Lambda$. 2) SIF fiber with the effective cladding index of a PCF, $n_{\mathrm{co}}=n_{\mathrm{si}} \rho=\Lambda$. 3) PCF with $n_{c o}=n_{s i} \rho=0.58 \Lambda$. 4) PCF with a core-index equal to a honeycomb-index, $\rho=\Lambda$. The inset shows the similar properties of the second order mode.

The last curves show the result of using the effective honeycomb index as the core index and a core radius of $\Lambda$. Now the second order mode cut-off has decreased to 3.3, which is closer to the result of a SIF, albeit still too large a value. However, $\mathrm{B}$ is approximately 0.93 at the second order mode cut-off, which is significantly larger than the value obtained by the considered SIF approximation ( $B$ is less than 0.6). As expected the $B$ values approach 1 for large $\mathrm{V}_{\text {eff }}$-values. Notice that the maximum B-value of the SIF fibre $(0.96)$ lies between the maximum B-values found from the two different PCF core assumptions $(0.88$ and $\mathrm{I}$ respectively). This indicates that in order to create an effective index approach, which could accurately predict the mode-index of the guided modes as a function of frequency, one would have to employ not only a coreradius, $\rho$, less than $\Lambda$, but also an effective core-index below the refractive index of silica. The specific values of core-radius and refractive index would furthermore be likely to be wavelength dependent.

We deduce that the honeycomb index underestimates the effective refractive index of the core-region, while silica overestimates the core-index. Both models predict that the effective core-radius is less than $\Lambda$.

This result is not really surprising! One cannot expect to be able to accurately investigate a PCF with a rich topology using a simple step index fibre approach. However, the effective index approach is able to provide insight into basic physical properties of PCFs. It correctly identifies the possibility of PCFs that are singlemode at all wavelengths. Correspondingly it predicts that PCFs have increasing bending losses at short wavelengths. Effective index approaches also correctly predict that very small effective mode-areas are possible using PCFs. However, accurate modelling of PCFs with an effective index approach appears to demand that one is able to predict the actual core-index and core-radius as a function of wavelength, a task which at present only seems possible using retrofitting schemes, which of cource is no real solution at all.

\section{Conclusion:}

We have found that not only the cladding region, but also the core-region of Photonic Crystal Fibres (PCFs) has an effective index that varies with frequency. We conclude that it is not possible to accurately model PCFs using an effective index model. Instead, models that accurately describe the topology of PCFs must be employed. Still, used with care, an effective index approach can provide many qualitative answers concerning the basic behaviour of PCFs.

\section{references}

/1/ Bjarklev A. et. al. OFC 2001, TuCl

12) Broderick N. et. al. Optics Letters, 16, 1999, p. $1395-$ 97

13/ Knight J. C. et. al. Photonics Technology Letters, 12, 2000, p. 807-809

14/ Birks T.A. et. al. OFC 1999, FG4.

15/ Johnson S. et. al. Optics Express, 8, 2001, p. 173-90.

16/ Monro, T..M. et. al. J. Lightwave Technology, 17, no. 6, 1999, p. 1093-1102.

$17 /$ Brechet F. et. al. Optical Fiber Technology, 6, 2000, p. 181-91.

18/ Ghosh R. et. al. Electronics Letters, 35, no. 21, 1999, p. 1873-75.

19/ Silvestre E. et. al. J. Lightwave Technology, 16, 1998, p. 923-28.

/10/ Agrawal G. P., Fiber-Optic Communication Systems, 1997, Wiley \& Sons. 